Rafidain Journal of Science

Vol. 30, No. 1, pp.1-10, 2021

DOI: 10.33899/rjs.2021.167677

https://rsci.mosuljournals.com

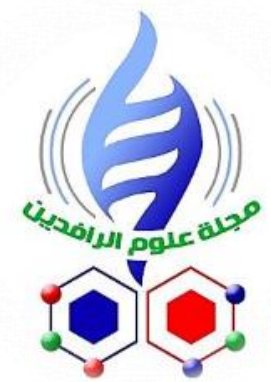

ISSN: 1608-9391

e-ISSN: 2664-2786

Received:6/9/2020

Accepted:11/10/2020

\section{Variation of Scale Factor and Deceleration Parameter Through the Cosmic Evolution of Time and Temperature}

\author{
*Ahmed S. Ali \\ **Mohammed K. Zeki \\ Department of Physics/ College of Science/ University of Mosul \\ "E-mail: ahmedsubhi2226@gmail.com \\ **E-mail: mkzkanz@yahoo.com
}

\begin{abstract}
In this work the differential Friedmann-Lemaître-RobertsonWalker model solutions which offer different cosmological parameters. We studied the effect of time and temperature evolution on these cosmological parameters, such as the scale factor $\mathrm{R}(\mathrm{t})$ and the deceleration parameter $\mathrm{q}$, across radiation, matter and dark energy eras. The nature of the variations in time and temperature of these quantities are consistent with that obtained from other cosmological models which have taken into account astrophysical observations. Time and temperature variations of these parameters have been shown graphically. These parameters provided strong evidence about the expansion and acceleration of the universe. The scale factor and the deceleration parameter show a linear change with the increase in time during the radiation and matter dominated eras, followed by a rapid expansion during the dark energy era, indicating that the universe has a transition from a phase of decelerated expansion to an accelerated expansion, which is contributed to dark energy. This led to a flat, homogeneous, and isotropic universe.
\end{abstract}

Keywords: Models of the universe, dark energy, cosmological parameters, cosmic acceleration. 


\section{INTRODUCTION}

Discovery of the cosmic acceleration was probably one of the most importance not only for modern cosmology but also for physics in general. However, in 1998 two independent collaborations (Riess et al., 1998; Perlmutter et al., 1999) having researched distant supernovae accelerated. It, presented convincing evidence of the fact that the expansion of universe is turned out that the brightness decreases in average considerably faster than it was commonly derived from the Big Bang, firmly confirmed that the universe is expanding with acceleration, it is now a widely accepted fact, that the universe has made a transition from a phase of decelerated expansion to its present phase of accelerated expansion.

It became also clear that our universe contains about $4 \%$ of ordinary matter, $23 \%$ of Dark Matter and $73 \%$ of Dark Energy. However, the nature of dark matter and dark Energy, as well as the cause of the accelerated expansion of our universe are still unknown is referred to as dark energy, and it is generally believed to be the sole cause of accelerated expansion of the universe. It has not yet been possible to determine its true nature. Recent study, based on an analysis of supernova data, by (Padmanabhan 2003), has shown that the deceleration parameter $(q)$ of the universe has certainly changed its sign from positive to negative, indicating a change of phase from deceleration to acceleration. The aim of the present work is to evaluate and test the scale factor $R(t)$ and the deceleration parameter $\mathrm{q}$, across radiation, matter and dark energy dominated eras.

\section{METHOD}

This section summarizes some basic concepts and descriptions of a cosmological model within the General Relativity system.

\section{Friedmann Equations}

Friedmann-Lemaître-Robertson-Walker model were introduced as a solution for Einstein's equations, (Weinberg, 1972).

$$
R_{\mu v}-\frac{1}{2} g_{\mu v} R+\Lambda g_{\mu v}=\frac{-8 \pi G}{c^{4}} T_{\mu v}
$$

Where, $\mathrm{R}_{\mu \nu}$ the Ricci tensor, $\mathrm{g}_{\mu \nu}$ the metric tensor, $\mathrm{R}$ the scalar curvature, $\mathrm{T}_{\mu \nu}$ the energy momentum-tensor, $\mathrm{G}$ the Newtonian gravitational constant, $\Lambda$ the cosmological constant and $\mathrm{c}$ the speed of light.

The Friedmann-Lemaître-Robertson-Walker metric (FLRW metric) describes the geometry in an homogeneous, isotropic universe and the Friedmann dynamic universe equations are (Weinberg 2008):

$$
\begin{aligned}
& \left(\frac{\dot{R}}{R}\right)^{2}+\frac{k c^{2}}{R^{2}}-\frac{\Lambda c^{2}}{3}=\frac{8 \pi G}{3} \rho \\
& \frac{2 \ddot{R}}{R}+\left(\frac{\ddot{R}}{R}\right)^{2}+\frac{k c^{2}}{R^{2}}+c^{2} \Lambda=\frac{-8 \pi G}{3} p
\end{aligned}
$$

$\mathrm{R}$ is the scale factor of the universe normalized to unity at the present time, $\frac{R}{R}$ represents Hubble's parameter (a dot represents time derivative), $\mathrm{k}$ is the curvature parameter of the universe given by $+1,0$, and -1 for positive, flat, and negative curvature respectively, $\rho$ is the energy density (energy per unit volume) of the universe and $\mathrm{p}$ is the pressure of the universe. 


\section{The Scale Factor of the Universe}

To find the R(t) scale factor, a link between the two equations (2) and (3) eliminates $\ddot{R}$ to get:

$$
\frac{\dot{R}^{2}(t)}{R^{2}(t)}-\frac{8 \pi G \rho}{3}=\frac{-K}{R^{2}(t)}
$$

Where $\mathrm{R}(\mathrm{t})$ is the scale factor of the universe, $\mathrm{G}$ is Newtonian gravitational constant and $\rho$ is the total energy density for the radiation $\left(\rho_{r}\right)$, matter $\left(\rho_{m}\right)$ and dark energy $\left(\rho_{d}\right)$ eras $\left(\rho=\rho_{r}+\rho_{m}+\rho_{d}\right)$, listed in the following formulae (Misner, 1973).

$$
\begin{aligned}
\rho_{\mathrm{r}} & =\frac{\mathrm{C}_{\mathrm{r}}}{\mathrm{R}^{4}(\mathrm{t})} \\
\rho_{\mathrm{m}} & =\frac{\mathrm{C}_{\mathrm{m}}}{\mathrm{R}^{3}(\mathrm{t})}=\frac{\mathrm{M}}{\mathrm{R}^{3}(\mathrm{t})} \\
\rho_{\mathrm{d}} & =\frac{\text { constant }}{\mathrm{V}^{\circ}}=\text { constant }=\rho_{0}
\end{aligned}
$$

Where $\mathrm{C}_{\mathrm{r}}=\mathrm{hc}$ ( $\mathrm{h}$ is the Plank's constant and $\mathrm{c}$ is the speed of light), $\mathrm{C}_{\mathrm{m}}=\mathrm{M}$ (the mass of the universe), $\mathrm{V}$ is the volume of the universe and $\rho_{0}=9.74 \times 10^{-30} \mathrm{gm} / \mathrm{cm}^{3}$, is the density of the present universe.

The solution of equation (4) gives the scale factor at the three different eras:

\section{The Scale Factor at the Radiation Dominated Era:}

The solution of Friedmann equations (4) in some simple cases, using a guess power law, setting

$$
\begin{gathered}
\frac{\mathrm{R}(\mathrm{t})}{\mathrm{R}\left(\mathrm{t}_{\mathrm{o}}\right)}=\left(\frac{\mathrm{t}}{\mathrm{t}_{\mathrm{o}}}\right)^{\alpha} \\
R(t)=\frac{R\left(\mathrm{t}_{\mathrm{o}}\right)}{\mathrm{t}_{\mathrm{o}}{ }^{\sigma}} t^{\sigma}=\mathrm{C} t^{\sigma}
\end{gathered}
$$

Where $C=\frac{R\left(\mathrm{t}_{0}\right)}{\mathrm{t}_{0}{ }^{\alpha}}$ and $\alpha$ are constants.

Combining equation (4), (5) and (8), with $\mathrm{k}=0$ (flat universe), yields

$$
\mathrm{R}_{\mathrm{r}}(\mathrm{t})=\sqrt[4]{\frac{32 \pi \mathrm{Gh}}{3 \mathrm{C}}} \mathrm{t}^{1 / 2}
$$

Which shows that the scale factor at radiation era $R_{r}(t)$ is proportional to the time of power half.

\section{The Scale Factor at the Matter Dominated Era:}

The same procedure can be used to find the scale factor at the dominated era of matter by combining equations (4), (6) and (8) to get:

$R_{m}(t)=\sqrt[g]{6 \pi G M} t^{2 / 3}$

Here $R_{m}(t)$ is proportional to the time of power $(2 / 3)$.

The Scale Factor at the Dark Energy Dominated Era:

In this case $\rho_{d}=\rho_{o}$, so equation (4) becomes:

$R_{d}(\mathrm{t})=\mathrm{C}_{1} \mathrm{e}^{\mathrm{H}(\mathrm{t}) \mathrm{t}}$ 
Where $\mathrm{H}(\mathrm{t})$ is Hubble constant and $\mathrm{C}_{1}=R_{\mathrm{o}} \mathrm{e}^{-\mathrm{H}_{\mathrm{o}} \mathrm{t}_{\mathrm{o}}}$ (Weinberg 1972), equation (11) shows that the scale factor $\mathrm{R}(\mathrm{t})$ of the universe is rapidly exponentially extended describing an accelerated universe.

\section{Variation of the Deceleration Parameter}

Using the well-known definition of the deceleration parameter (Weinberg, 1972),

$$
q_{(t)}=q=-\frac{\ddot{R}(t)}{\dot{R}(t)} \frac{R(t)}{\dot{R}(t)}
$$

then the deceleration parameters in the three different eras are,

\section{Deceleration parameter at Radiation Dominated Era:}

Differentiate equation (9) twice with respect to time, then

$$
\dot{R}(t)=\frac{1}{2} \sqrt[4]{\frac{32 \pi G h}{3 C}} t^{-\frac{1}{2}}
$$

and

$$
\ddot{R}(t)=-\frac{1}{4} \sqrt[4]{\frac{32 \pi G h}{3 C}} t^{-\frac{\pi}{2}}
$$

A simple substitution of equations (9), (13) and (14) in (12) to get:

$$
q_{r}=+1
$$

\section{Deceleration Parameter at Matter Dominated Era:}

Similarly differentiates equation (10) twice with respect to time:

$$
\begin{aligned}
& \dot{R}(t)=\frac{2}{3} \sqrt[s]{6 \pi G M} t^{-1 / 3} \\
& \dot{R}(t)=\frac{-2}{9} \sqrt[s]{6 \pi G M} t^{-2 / 3}
\end{aligned}
$$

by direct substitution, equation (12) reads:

$$
q_{m}=\frac{1}{2}
$$

It is obvious that $\mathrm{q}_{\mathrm{r}}$ and $\mathrm{q}_{\mathrm{m}}$ show a decrease in their values respectively, indicating that in these two successive eras the universe is decelerated.

\section{Deceleration Parameter at Dark Energy Dominated Era:}

Finally, the value of $q_{d}$ can be found by substituting equation (11) and their first and the second derivative into equation (12) yields:

$$
q_{d}=-1
$$

The negative value of $q_{d}$ indicate acceleration of the universe.

\section{RESULTS}

It should be noted at the beginning firstly that each figure was divided into three parts, shape A represented the radiation era, shape B represented the matter era, while shape C includes the radiation, matter and dark energy eras. Secondly figure was reversed (for temperature dependence) due to the MATLAB coordinates rearrangement. 
Fig.(1) shows that the scale universe is so small in the early universe with a huge temperature value, as time goes by, the temperature steadily decreases, such that the temperature at the present time $13.8 \mathrm{Gyrs}$ (the exponential value of $\approx 23.3$ at the time axis), is about $2.71 \mathrm{~K}$ (the exponential value of $\approx 0.9$ at the temperature axis), this value is consistent with astrophysical observation of cosmic microwave background temperature of the universe $\mathrm{T} \approx 2.71 \mathrm{~K}$ (Durrer, 2008).

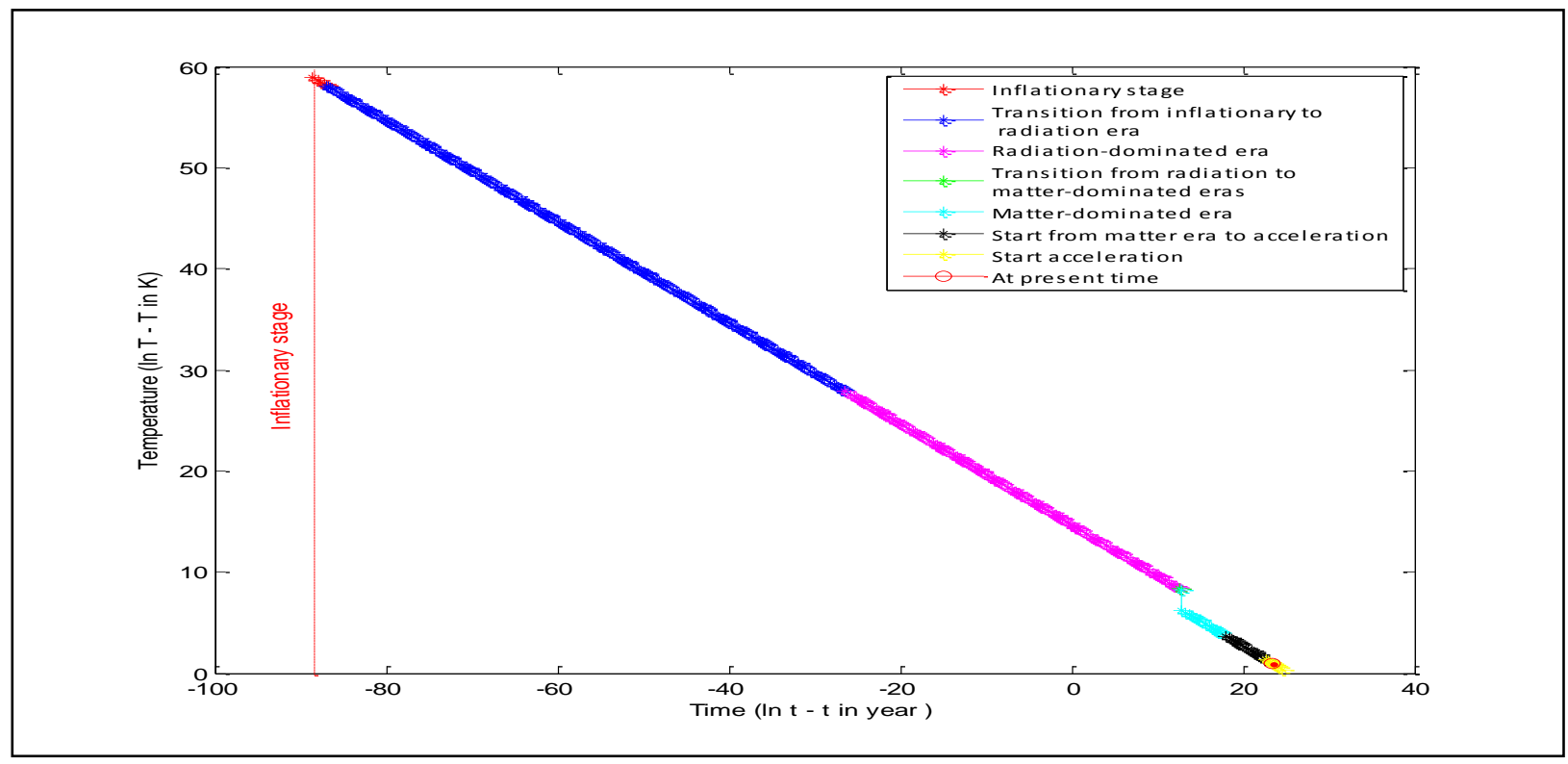

Fig. 1 : Evolution of time and temperature.

Fig. (2) shows variations in the scale factor (equations 9,10 and 11) with the time and temperature in the three different dominated eras. Fig. (2A) shows the variation of the scale factor as a function of time and time beyond. It increases with time, indicating clearly the expansion of the universe with time, It has been found that the universe radius (scale factor) at present time (13.8 Gyrs) is just the exponential value of $(62)$ at the vertical axis, i.e. $R\left(t_{o}\right)=8.8 \mathrm{E}+26$ meter. While scale factor in Fig. (2B) increases as the temperature decreases such that the temperature in the present universe radius is the horizontal axis exponential value of $(0.99), \mathrm{T}_{\text {present }}=2.69 \mathrm{~K}$. These results are consistent with the big-bang prediction that the universe's Cosmic Microwave Background (CMB) is equal to $2.71 \mathrm{~K}$ (Durrer, 2008). 


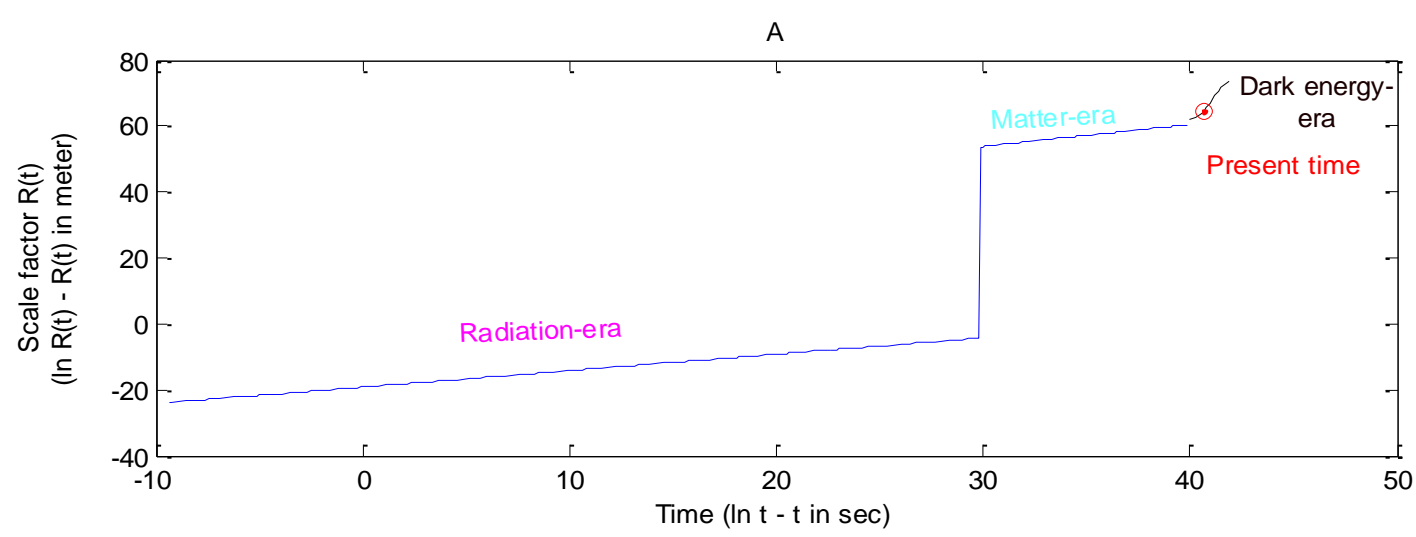

B

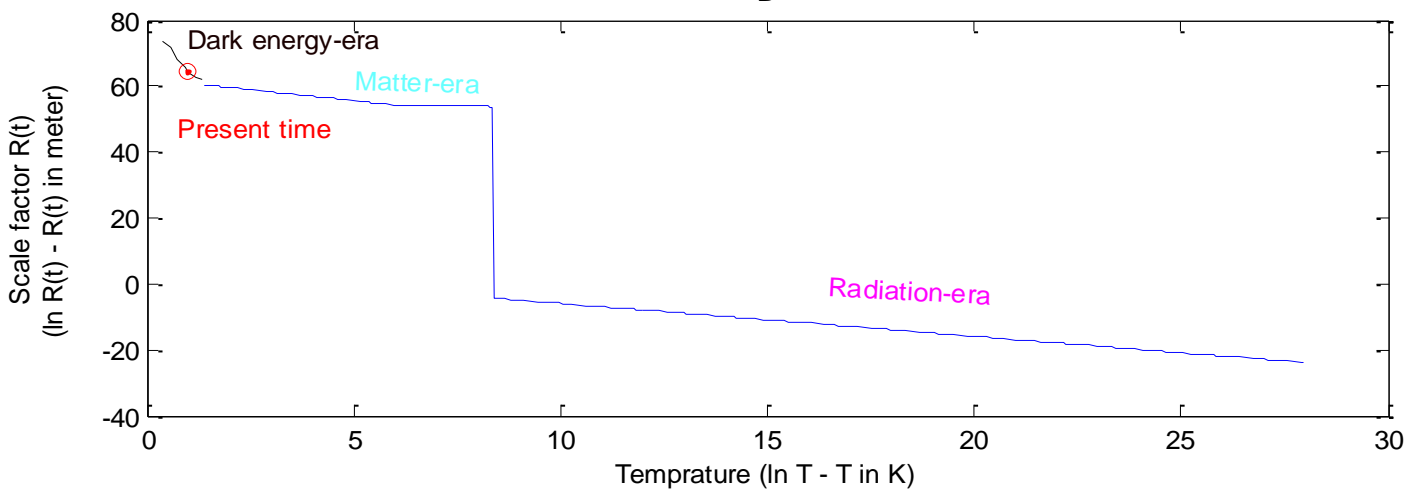

Fig. 2: Evolution of the scale factor as a function of time and temperature.

Fig. (3 and 4) show the variation of the deceleration parameter (equations 15, 18 and 19) as a function of time and temperature. They show a change of sign from positive in both radiation and matter dominated eras (Figs. 3A, 3B and 4A, 4B) to a negative value at the present time (Figs. 4c and $5 \mathrm{C}$ ), indicating a transition from a phase of decelerated expansion to accelerated expansion.

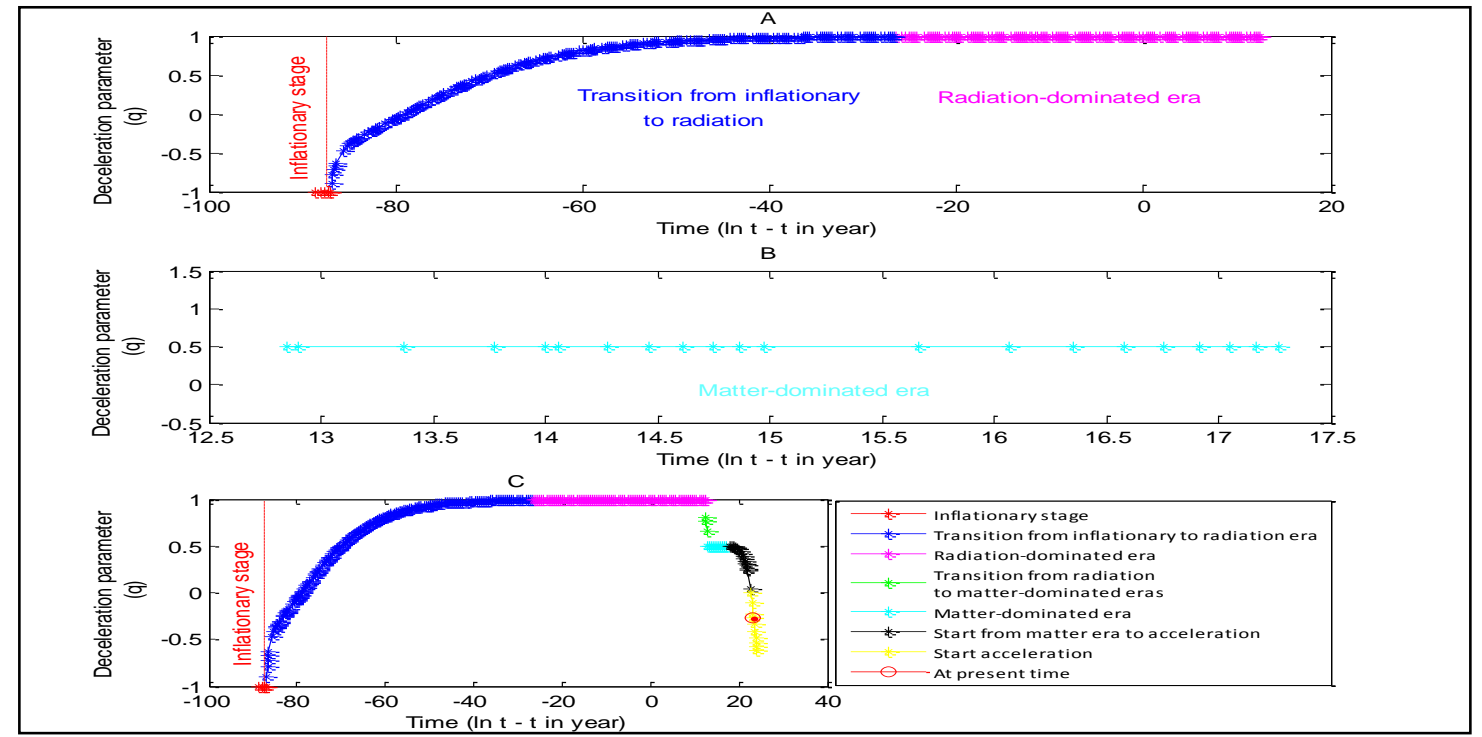

Fig. 3 : Evolution of the deceleration parameter with time. 


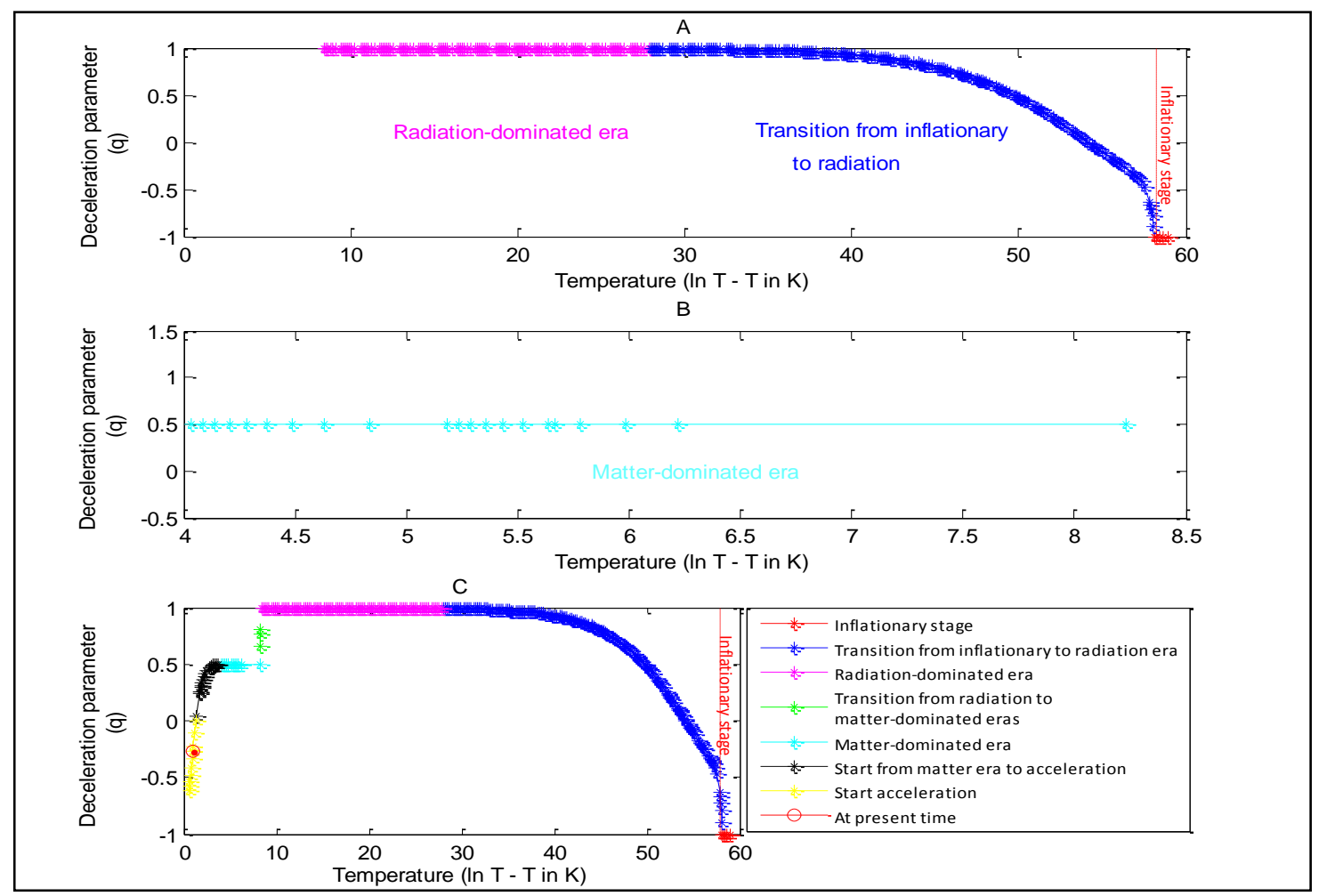

Fig. 4 : Evolution of the deceleration parameter with temperature.

Fig. 5 shows an increase in the scale factor that began with the transition from the inflationary to the radiation era, associated with a negative value of $\mathrm{q}$, which means there some how a negative pressure pushing out the contents of the universe which makes a pre-expansion. The value of $\mathrm{q}$ become +1 at the radiation era, then to decreases gradually to half at the matter dominated era, this indicating that the universe begins to decelerate. What unexpected happened that at the end of the matter the value of the deceleration parameters turned into a negative value, suggesting that the universe began to accelerate again now and time beyond. 


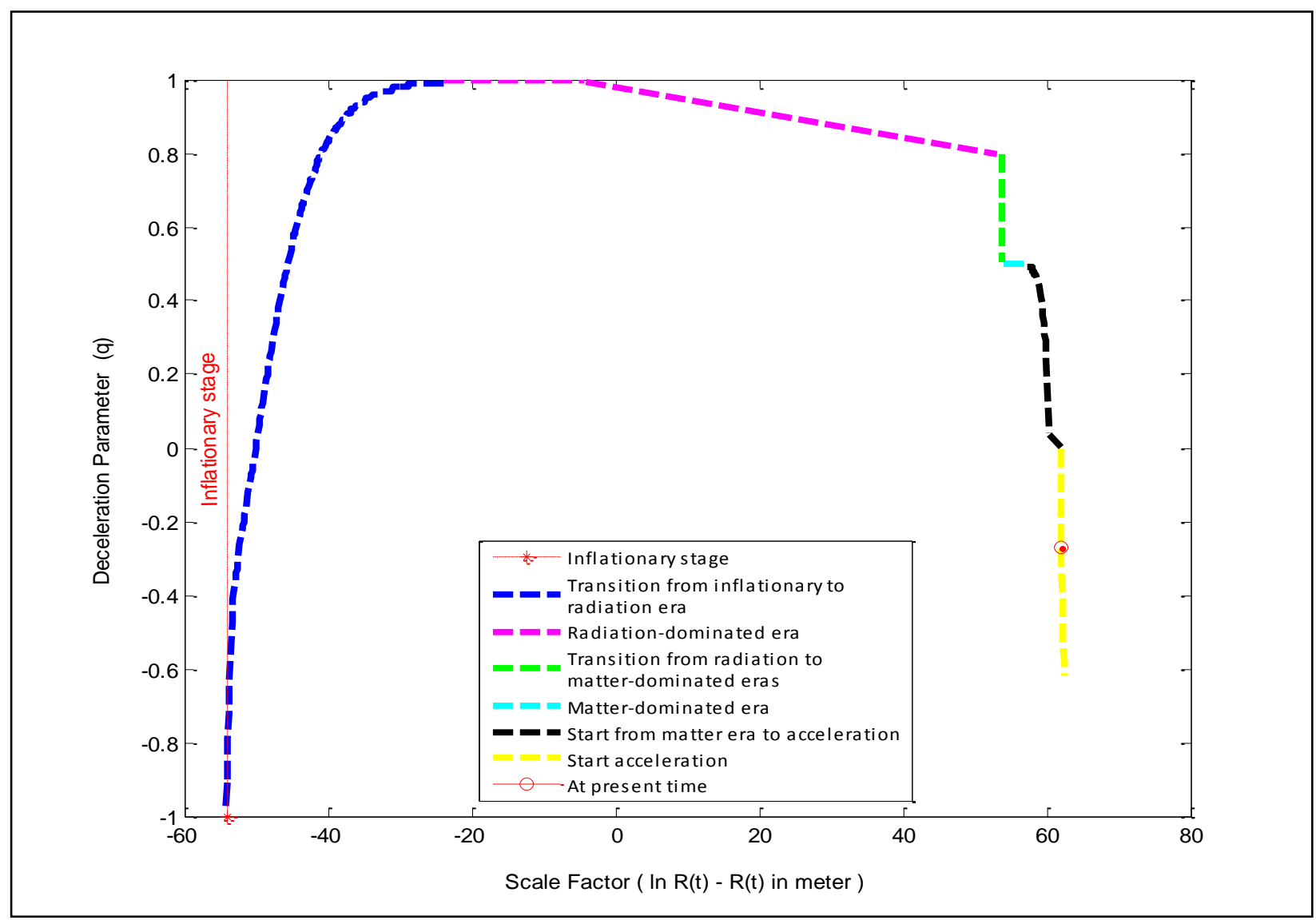

Fig. 5: Variation of the deceleration parameter with the scale factor

\section{DISCUSSION}

In this work Friedmann-Lemaître-Robertson-Walker model were introduced as a solution for Einstein's equations as a homogeneous and isotropic universe. we have studied the time evolution of two cosmological parameters the scale factor and deceleration parameter as given in (Table 1) (Cahill, 2016; Crevecoeur, 2017). The time and temperature dependency were determined and shown graphically.

The time variation of the scale factor $R(t)$ shown in Fig. (2A) shows an increase in $R(t)$ in all eras, which clearly indicates the expansion of the universe, reaching the radius $(8.81 \mathrm{E}+26 \mathrm{~meter})$ at the present time

$\mathrm{t}=13.8 \mathrm{Gyr}$, while the scale factor in Fig. (2B) is inversely proportional to temperature, such that the current value of $\mathrm{R}(\mathrm{t})$ shows a temperature $\mathrm{T}_{\text {present }}=2.69 \mathrm{~K}$. These results are consistent with the big-bang prediction that the universe's Cosmic Microwave Background (CMB) which is equal to $2.71 \mathrm{~K}$ (Durrer, 2008).

The deceleration parameter variation with time, temperature and the scale factor Figs. (3-5) show a change of sign from positive ( 1 and 0.5 respectively at radiation and matter dominated eras) to negative value ( -0.27 now and -0.33 later on) indicating a transition from a phase of decelerated expansion at both radiation and matter dominated eras to accelerated expansion now and time beyond, (Bengochea, 2019; Roy et al., 2019; Lin and Qian, 2020).

Table 1: Parametric sample values during cosmological evolution (Crevecoeur, 2017)

\begin{tabular}{|c|c|c|c|c|c|}
\hline Eras & Radiation & Matter & Dark & Now & Beyond \\
\hline Time (year) & $3.17 \mathrm{E}-12$ & $3.79 \mathrm{E}+05$ & $7.12 \mathrm{E}+09$ & $1.38 \mathrm{E}+10$ & $1.58 \mathrm{E}+10$ \\
\hline Temperature (K) & $1.30 \mathrm{E}+12$ & $3.76 \mathrm{E}+03$ & $3.76 \mathrm{E}+00$ & $2.70 \mathrm{E}+00$ & $2.52 \mathrm{E}+00$ \\
\hline Scale factor R(t) (meter) & $4.59 \mathrm{E}-11$ & $2.63 \mathrm{E}+23$ & $7.66 \mathrm{E}+26$ & $8.81 \mathrm{E}+26$ & $9.17 \mathrm{E}+26$ \\
\hline Deceleration parameter q & 1.00 & 0.50 & 0 & -0.27 & -0.33 \\
\hline
\end{tabular}




\section{CONCLUSIONS}

Our conclusion based on three significant points. First, the energy density is inversely proportional to the increase in the scale factor showing a steady decrease in its value in both the eras of radiation and matter (equations 5 and 6), reaching its present constant value $\rho_{d}=\rho_{0}=9.74 \times 10^{-30}$ $\mathrm{gm} / \mathrm{cm}^{3}$ (equation 7), which means that the present universe is flat and homogenous.

Second its cleared that as time goes on, the scale factor $\mathrm{R}(\mathrm{t})$ increases as a step function, in both radiation and matter dominated eras Fig. (2A), then shows a rapid expansion in the dark energy era, on the other hand it increases as temperature decrease Fig. (2B), reaching its present value $8.81 \mathrm{E}+26 \mathrm{~cm}$, at $\mathrm{T}_{\text {present }}=2.69 \mathrm{~K}$ which is consistent with cosmic microwave background temperature of the universe $\mathrm{T} \approx 2.71 \mathrm{~K}$ (Durrer, 2008). Finally, the deceleration parameter variance with time and temperature (Figs. 3 and 4 ) indicate a change of sign from positive ( 1 and 0.5 at both radiation and matter dominated eras respectively) to negative value $(-0.27$ now and -0.33 time after), suggesting a transition from a decelerated expansion at both radiation and matter dominated eras to an accelerated expansion now and time beyond.

\section{REFERENCES}

Bengochea, G.R. (2019). What do we talk about when we speak of cosmological redshift? Revista Mexicana de Física E, 65(1), 22.

Cahill, K. (2016). The eras of radiation, matter, and dark energy: new information from the Planck Collaboration. Physics. arXiv:1606.08865v1(pop-ph).

Crevecoeur, G.U. (2017). Time evolution of dark energy and other cosmological parameters. Physics Essays, 30(3), 255-263.

Durrer, R. (2008). "The Cosmic Microwave Background”. Cambridge University Press.

Lin, K.; Qian, W.L. (2020). Cosmic evolution of dark energy in a generalized Rastall gravity. The European Physical J. C. 80(561).

Misner, C.W.; Thorne, K.J.; Wheeler, J.A. (1973). “Gravitation”. W.H. Freeman and Company Limited.

Padmanabhan, T.; Choudhury, T.R. (2003). A theoretician's analysis of the supernova data and the limitations in determining the nature of dark energy. Monthly Notices of the Royal Astronom. Soc., 344(3), 823-834.

Perlmutter, S.; Aldering, G.; Goldhaber, G.; Knop, R.A.; Nugent, P.; Castro, P.G.; Deustua, S.; Fabbro, S.; Goobar, A.; Groom, D.E.; Hook, I.M.; Kim, A.G.; Kim, M.Y.; Lee, J.C. ; Nunes, N.J.; Pain, R.; Pennypacker, C.R.; Quimby, R.; Lidman, C.; ... Project, T. S. C. (1999). Measurements of $\Omega$ and $\Lambda$ from 42 High-Redshift Supernovae. The Astrophys. J., 517(2), 565-586.

Riess, A.G.; Filippenko, A.V.; Challis, P.; Clocchiatti, A.; Diercks, A.; Garnavich, P.M.; Gilliland, R.L.; Hogan, C.J.; Jha, S.; Kirshner, R.P.; Leibundgut, B.; Phillips, M.M.; Reiss, D.; Schmidt, B. P.; Schommer, R.A.; Smith, R.C.; Spyromilio, J.; Stubbs, C.; Suntzeff, N. B.; Tonry, J. (1998). Observational evidence from supernovae for an accelerating universe and a cosmological constant. The Astronom. J., 116(3), 1009-1038.

Roy, S.; Sarkar, A.; Ghosh, P. (2019). Time dependence of various cosmological parameters in the framework of Kaluza-Klein Space-Time. International J. Scientific Research in Sci. and Technol., 211-220, L., and Ratra, B. (2006). Cosmological constraints from Hubble Parameter versus redshift data. The Astrophys. J., 650(1), L5-L8.

Samushia, L.; Ratra, B. (2006). Cosmological constraints from Hubble Parameter versus redshift data. The Astrophys. J., 650(1), L5-L8.

Susskind, L. (2009). "Documents". (Stanford University Press).

Vishkarma, R.G.; Beesham, A. (1999). RW models with variable G and constant active gravitational mass. Nuovo Cimento B, 114B(6), 631-634.

Weinberg, S. (1972). "Gravitation and Cosmology”. John Wiley and Sons Inc.; (New York).

Weinberg, S. (2008). "Cosmology”. (Oxford University Press). 


\section{تباين عامل القياس ومعامل التباطؤ من خلال التطور الكوني للزمن ودرجة الحرارة}

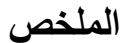

تضمن البحث استخدام حلول نموذج فريدمان- لوميتري- روبرتسون - ووكر التفاضلي للحصول على معاملات كونية

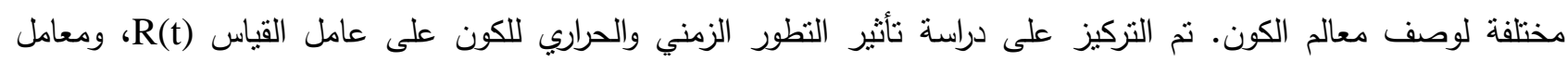
التباطؤ q خلال الفترات الزمنية للأحقاب الثلاثة الإشعاعية والمادية والطاقة المظلمة. وكانت طبيعة النتائج التي نم الحصول

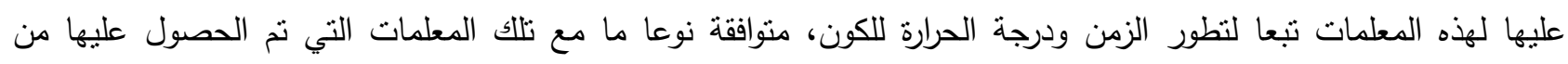

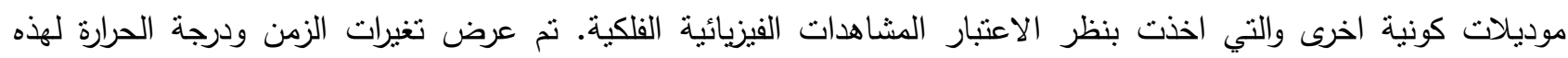
المعلمات بيانياً. قدمت المعلمات دليلًا قويًا على تمدد الكون وتسارعه، حيث اظهر كليه لخدئ من عامل القياس ومعامل التباطؤ تغيرا

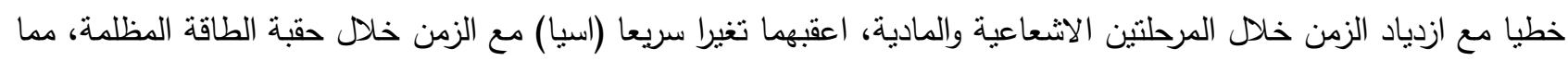

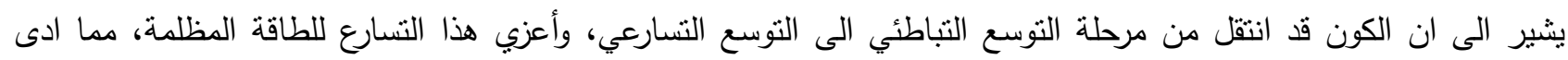
للحصول على كون منبسط ومتجانس الكثافة ومنسق الاتجاهات (منتاظر الخواص). الكلمات الدالة: الموديلات الكونية، الطاقة المظلمة، المعلمات الكونية، التسارع الكوني. 